\title{
CALIFORNIA's VISION FOR REACHING ZERO-CARBON EMISSIONS
}

PREPRINT

\author{
P. A. Sánchez-Pérez \\ University of California, Merced \\ Merced, CA, USA \\ pesapsanchez@gmail.com
}

\author{
S. Kurtz \\ University of California, Merced \\ Merced, CA, USA \\ skurtz@ucmerced.edu
}

June 5, 2020

\begin{abstract}
In "The 100 Percent Clean Energy Act of 2018" the California legislature set a target of $100 \%$ of California's electricity generated from renewable and zero-carbon sources by 2045 . The California Public Utility Commission (CPUC) and other state entities now have the task of planning to meet that target. The California Energy Commission (CEC) has sponsored multiple studies and sought public input on the pathways that they are exploring. A key result of that planning process is a Reference System Portfolio (RSP) based on existing and planned electricity generating capabilities, and modeled grid build out to meet the planned targets by 2045 at the lowest cost. Although this RSP has been discussed by the CEC in a public forum, to our knowledge, it has not been presented to the photovoltaic community. Here we document the CEC's current RSP, with emphasis on understanding their expectations for build out of solar as well as the associated need for storage and curtailment.
\end{abstract}

Keywords: Zero-carbon, electrical grid, storage, solar photovoltaic

\section{Introduction}

"The 100 Percent Clean Energy Act of 2018" [1] describes a series of targets for the deployment of renewable electricity generating plants on the pathway to the 2045 target of $100 \%$ renewable and zero-carbon electricity from California's electricity grid. While California was one of the first to set such targets, there are now multiple states and utilities across the United States as well as countries around the world that are making similar commitments. Maximum output of California's utility-scale solar PV was over $11 \mathrm{GW}$ in 2019 [2] — ranking number 1 in both operational capacity and generation for the U.S [3]. In 2019, of the electricity generated in California, the Energy Information Agency (EIA) estimates that $20 \%$ was generated by solar systems. Thus, California is well positioned to be a leader in the adoption of solar energy and in planning for reaching zero-carbon electricity. Others working toward adoption of solar and other renewable energy technologies may find California's planning to be informative.

The CEC issues a biennial report entitled "Integrated Energy Policy Report" (IEPR) which includes a forecast of the anticipated electricity demand for California. The CPUC uses this forecast as part of its Integrated Resource Planning (IRP) as a basis for how California can meet its clean-energy targets [6]. This planning is implemented in the Reference System Portfolio (RSP) that is then used as a baseline to explore the effects of policy actions that the CPUC may take. The RSP is a living document that is updated to reflect new results from the IEPR and the IRP.

In this work, we analyze the RSP and how it could impact the big picture of the solar industry. We start by describing some of the assumptions made by the RSP. We then summarize the RSP's vision for what the grid will be like in 2045, especially considering the role of solar plus storage in reaching the zero-carbon target. 
JUNE 5, 2020

\section{General Assumptions of RESOLVE}

The RSP is implemented using the capacity-expansion model RESOLVE [5] to optimize (minimize) the total cost of the electrical grid while meeting multiple constraints. The full model is written in Python and is open-source. All the inputs and results of the optimization model for the RSP are provided online by the CPUC. RESOLVE has 54 input files to guide the simulation, which the CPUC explained in [4]. It systematically works through the loading of the data, defines how to calculate the relevant parameters, defines the constraints, and then defines the objective function which includes the costs of installing and operating the grid, including operations and maintenance costs, fuel costs, costs for ancillary services, and any penalties. Finally, the results are summarized in almost 40 files.

The current RSP is descriptively labeled as: 46MMT_20200207_2045_2GWPRM_NOOTCEXT_RSP_PD. This scenario targets reaching 46 million-metric-tons of eq- $\mathrm{gCO}_{2}$ emissions by 2045 . It also requires a planning reserve margin of $2 \mathrm{GW}$ and assumes that the date for shutting down some once-through-cooling power plants by the end of 2024 will not be extended.

The model is spatially resolved in seven (7) balancing zones with five (5) zones capturing California balancing authorities and two zones that represent regional aggregations of out-of-state balancing authorities [4] as shown in Fig 1 . The model performs load balancing, provides planning reserve and applies operational constraints. Input files define 171 electricity generating resources, each with associated cost, starting capacity, guidance about increasing or retiring that capacity, ability to provide ancillary services, zone location, constraints on rate of ramping output, etc. The model gains computational speed by limiting the simulation to 37 days weighted to reflect statistics for a typical year with respect to frequency distributions for load, solar generation and wind generation. The results of the optimization are strongly guided by the inputs, which include many constraints, such as:

- The model only allows building new power plants in the CAISO zone;

- The policy targets are applied only for the CAISO zone;

- Some nuclear and coal resources are retired in 2025;

- Build out of solar, wind, geothermal and biomass is limited to practical values for each location;

- Only renewable technology (solar PV and wind) can be curtailed;

- Each of the 37 days is modeled to loop back into itself, which does not provide a mechanism for seeing the effect of having a sunny day followed by a cloudy day or other weather patterns that might result in multi-day storage of electricity;

- Costs of installing and operating plants are defined according to the technology and year of installation with added costs defined for fuel consumption and penalties.

\section{Methodology}

For this work, we analyzed the results published by the CEC for the RSP scenario. The first metric we calculated was the total operational capacity by each period in the RSP. The data required are in the file named resource_build.csv. This file contains a summary of the resulting capacity expansion with information such as the resource, technology, planned, operational, and cumulative capacity. We aggregated the "operational_capacity_mw" column by period and technology and added the total operational capacity for all the balancing zones and CAISO only. Additionally, we classified each technology from the input file into a broader technology category to simplify the visuals.

Next, we calculated the total electricity generation for each period, including storage discharge and curtailed electricity. To create this dataset, we used two separate files: operations.csv that contains the resulting operational dispatch (including storage charge and discharge) and curtailment.csv that has detailed information about the curtailment. For this metric, we aggregated the "power_mw" column by period and technology group for all the zones and for CAISO only. For the curtailed electricity, we added the columns: "scheduled_curtailment" and "sub_hourly_curtailment" and then added it to the top of the total electricity generation.

Finally, we analyzed four different curtailment scenarios. We selected four (4) different days by considering the curtailment shape and that we had one day per season of the year to show different generation/curtailment profiles. The selected days for each season are:

- Spring: Day $20(5 / 7 / 2008)$

- Summer: Day 36 (8/17/2009)

- Autumn: Day 24 (10/28/2008) 
JUNE 5, 2020

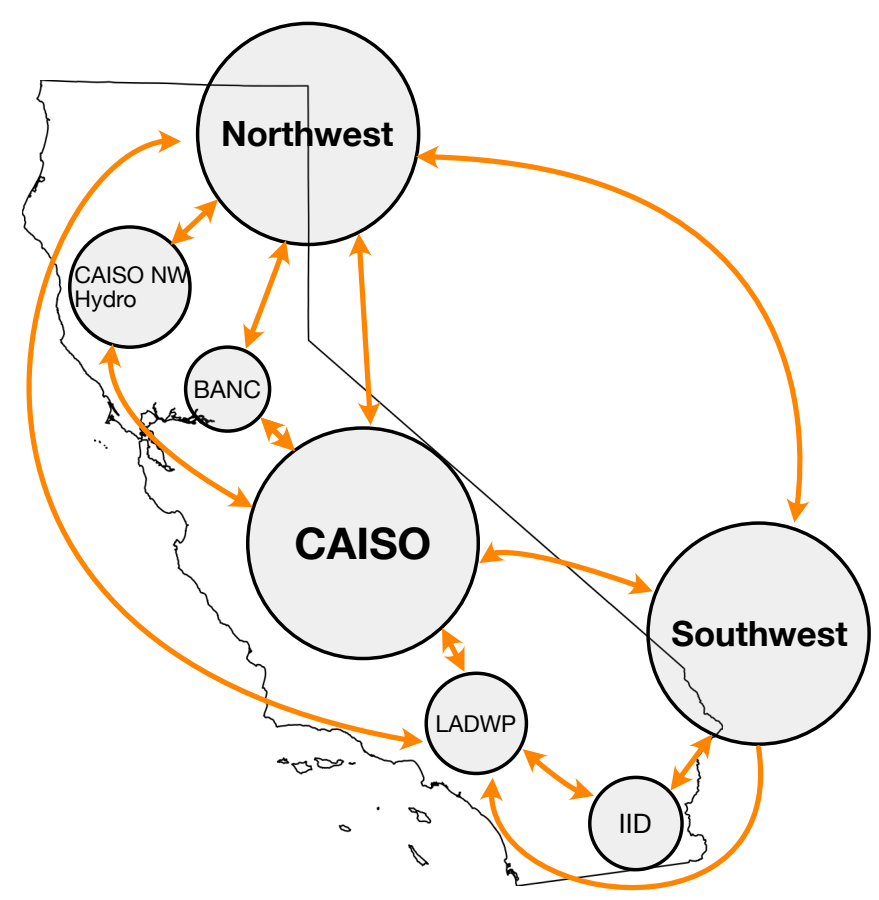

Figure 1: Schematic diagram of California showing the balancing zones considered in RESOLVE. Arrows show considered transmission between zones.

- Winter: Day $28(1 / 21 / 2009)$

For each day, we took the load profile ("input_load_mw"), the battery charging ("storage_charging_mw"), and the total generation ("total_generation_mw") from the file loads_and_power_balance.csv and we aggregated them by "timepoint_id" just for the CAISO zone. Then, we calculated the net imports by subtracting the exports "unspecified_power_negative_direction" from the imports "unspecified_power_positive_direction" from the file transmit_power.csv. Lastly, we did the same procedure for the curtailed electricity as mentioned above but aggregating it by "timepoint_id".

\section{Results and discussion}

Fig. 2 2 shows the total operational capacity for all the periods simulated, wherein Fig. 2a has the total capacity for all the balancing zones and Fig. 2bjust for CAISO (including CAISO's hydro). In 2045 (the target for reaching "zero carbon" emissions) solar PV technology comprises about $52 \%$ and about $37 \%$ of the operational capacity for CAISO and for all the zones, respectively. Wind plants comprise only about $8 \%$ of the total, reflecting build out to nearly $100 \%$ of the capacity limit specified by the assumptions of the RSP. Hydropower plays an important role as a regional source of electricity, with about $13 \%$ of the capacity for all zones. Biomass and geothermal capacities are small $(<1.5 \%)$. The last nuclear plant in CAISO is planned to be retired in 2025, while a small amount of nuclear power is retained in the zones outside of CAISO. The implementation of storage is linked to the increase in deployment of solar, comprising about $25 \%$ and $16 \%$ of the electricity generating capacity in CAISO and in all zones, respectively. Fossil-fuel-based technologies decrease in the share of the energy portfolio for California, with all coal plants planned to retire by 2025. Fossil fuels play a larger role outside of CAISO (see Fig. 2b), reflecting that the RSP is designed to reach zero carbon emissions within CAISO and does not attempt to optimize the energy mix outside of CAISO, where the CPUC has less control.

Fig. 3 shows the resulting total electricity generation for each period. From Fig. 3 we can summarize the following: Solar is the main supply of electricity for CAISO in 2045 followed by storage. The roles of geothermal, coal and hydropower increase relative to their role in the capacity build out, reflecting their higher capacity factors relative to solar, storage and natural gas. Curtailed electricity increases up to about $7 \%$ of the total electricity or about $13 \%$ of the solar and wind generation. Note that the total load for 2045 was $381 \mathrm{TWh}$ and $883 \mathrm{TWh}$ for CAISO and for all the zones respectively. The total load is less than the height of the 2045 bars in 4 because we have included re-generation from storage and curtailment. 
JUNE 5, 2020

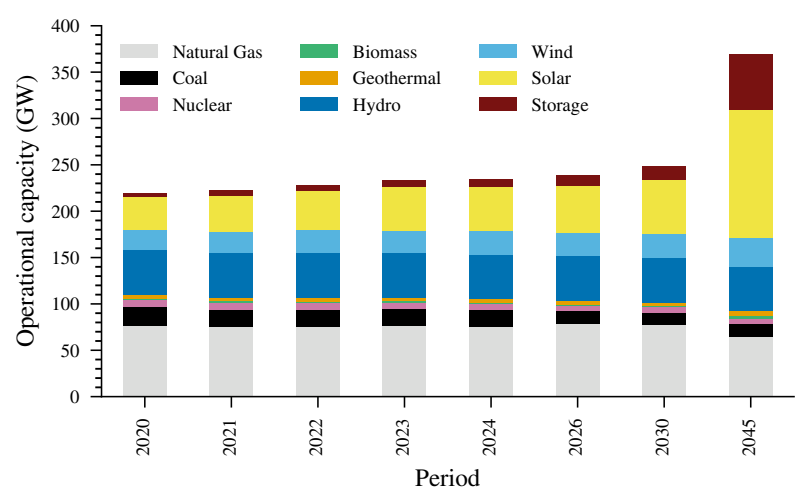

(a) All zones

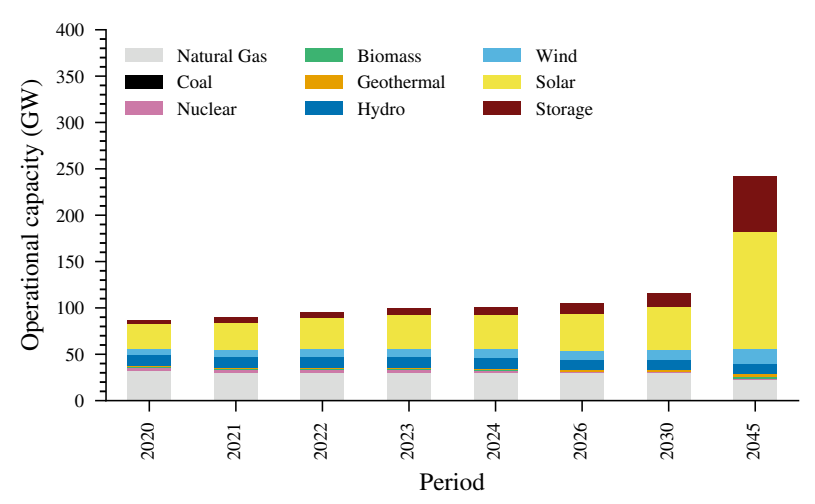

(b) CAISO

Figure 2: Operational capacity from the baseline scenario. Fig. 2a for all balancing zones and Fig. 2b for CAISO (including NW hydro) only. The spacing of the periods is irregular, with the seemingly spectacular growth in the last period reflecting 15 years of growth.

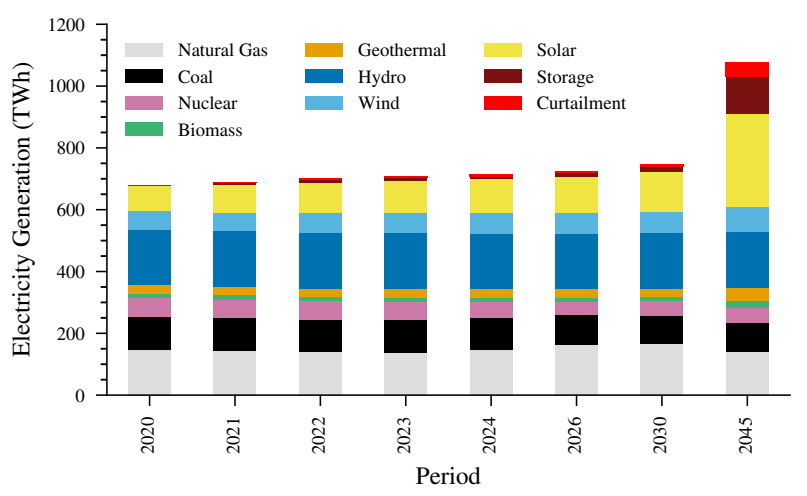

(a) All zones

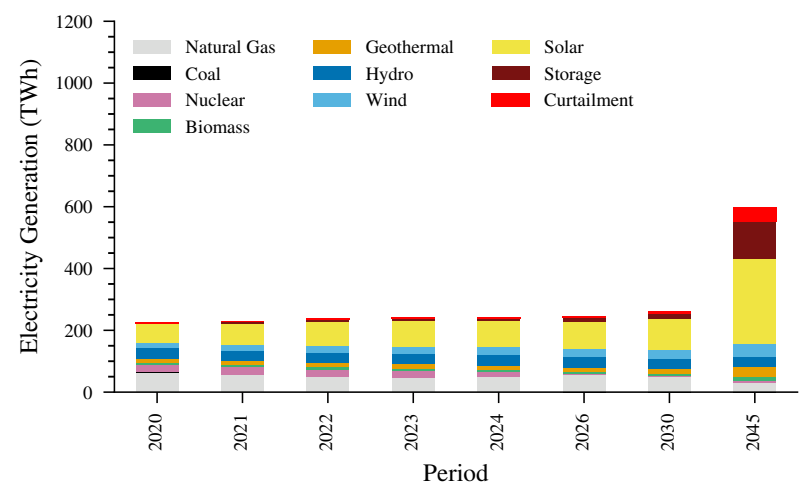

(b) CAISO

Figure 3: Electricity generation from the baseline scenario. Electricity re-generated from the batteries is included. Fig. 3a for all balancing zones and Fig. 3b for CAISO (including NW hydro) only. The spacing of the periods is irregular, with the seemingly spectacular growth in the last period reflecting 15 years of growth.

If off-shore wind or other source of wind power were to be used, solar energy and storage might be needed in a much smaller way.

Though the model sets the zero-carbon emission goal only for the CAISO zone, the adjacent zones play an important role in supplying electricity to CAISO. We anticipate that the RSP's vision for the adjacent zones may change as more utilities across the country (including some in adjacent states) are announcing plans to move toward zero-carbon emissions. The transitions of the other zones can be modeled in RESOLVE.

Fig. 4 shows the dispatch for the period 2045. We selected four days out of the 37 modeled days. These were chosen to exemplify the range of behaviors seen for the 37 days, ranging from days in which very little storage is used to days that use substantial storage, but no curtailment, to other days that use both storage and curtailment. During the winter, there are some days in which very little solar electricity is generated. On those days, storage is used very little. More commonly, solar electricity contributes more than half of the total electricity with some daytime electricity stored for nighttime use. For a more in-depth exploration of understanding the value of storage, see our companion paper in these proceedings [7].

\section{Conclusions}

California's current Reference System Portfolio proposes to reach zero-carbon electricity in 2045 with a very large build out of solar and storage. In the CAISO zone, the installed capacity in 2045 is modeled to include about 50\% solar, 

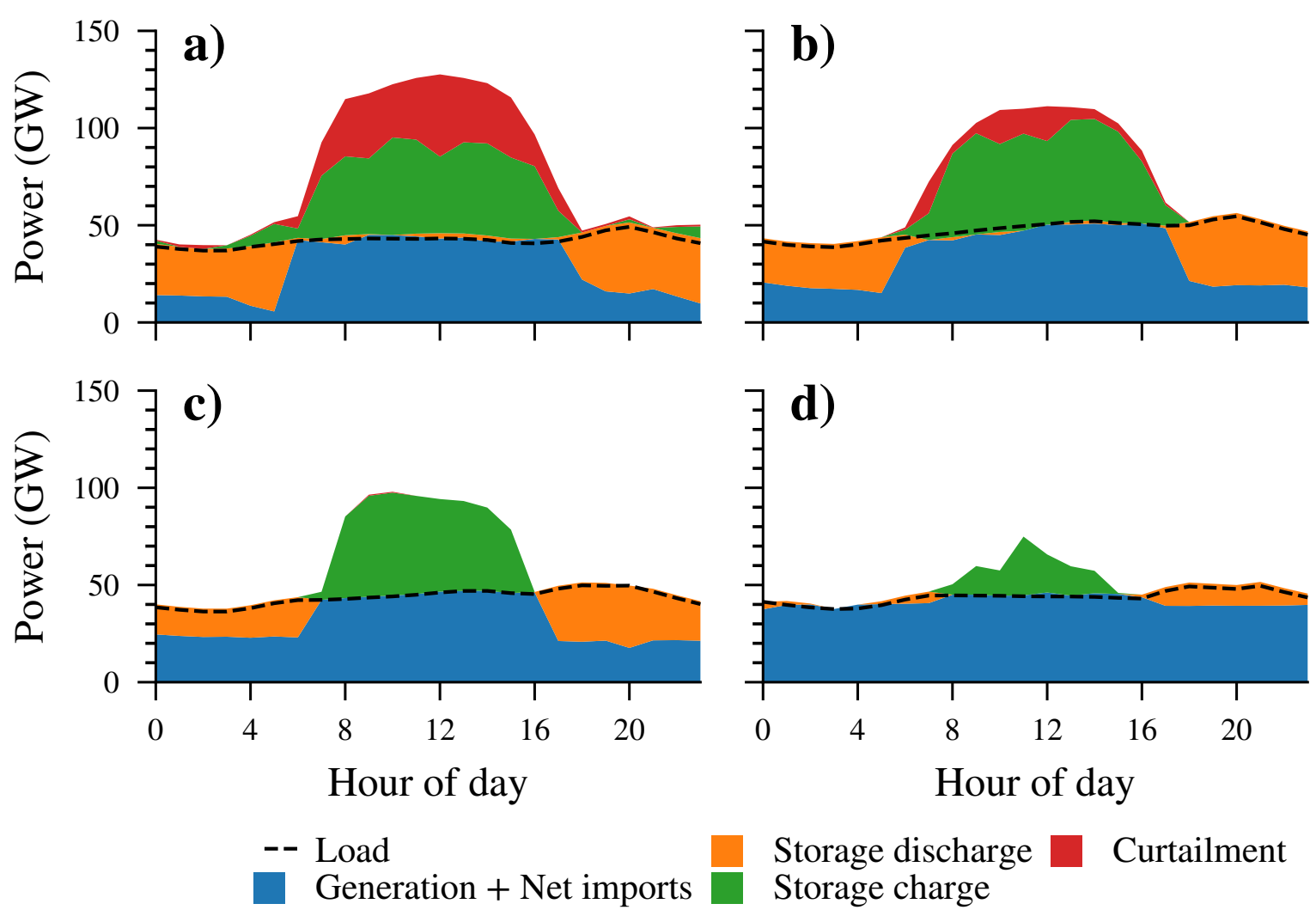

Figure 4: Resulting dispatch for four selected days. a) Spring day with high curtailment, b) Summer day with small curtailment, c) Autumn day, d) Winter day with low solar resource.

and about $25 \%$ storage and about $25 \%$ other generating technologies, when the breakout is calculated to include storage as one of the technologies in the capacity mix. The large build out of storage is required to provide electricity during the night when the sun isn't shining. In the CAISO zone, when the originally generated electricity is considered, $63 \%$ is modeled to be generated by solar, about $9 \%$ from wind, and $28 \%$ from other generating technologies; $27 \%$ of the load is found to be delivered after being stored in batteries. The relatively large build out of solar relative to wind is a result of assumed capacity limits for the wind generation, reflecting the limited wind resource in California. This large emphasis on solar is an opportunity for the solar industry to demonstrate its ability to provide more than $50 \%$ of the energy needed to run a grid. The curtailed electricity in the CAISO zone is about $14 \%$ of the electricity that is generated from solar and wind together.

We believe that as many places explore pathways to reach zero-carbon electricity grids, RESOLVE and similar models will be very useful toward identifying pathways. California being the state with the highest solar capacity installed serves as an excellent example. The optimization model used in the RSP was tailored for California, however, with appropriately revised inputs it may be adapted for use anywhere.

\section{Acknowledgment}

The authors thank University of California Merced undergraduate group Michele Campbell, Joseph Bello, and Jeremiah Tower for their useful work in developing the python scripts. We also thank Doug Condon, Rhonda Bailey, and Zabir Mahmud for useful comments.

\section{References}

[1] K. De León, “The 100 Percent Clean Energy Act of 2018 (SB-100)”. 2018. 
[2] California Independent System Operator, "Monthly Renewables Performance Report". Accessed: Jun. 01, 2020. [Online]. Available: https://bit.1y/2AHkMjS

[3] Energy Information Agency, "Electricity data browser". Accessed: Jun. 01, 2020. [Online]. Available: https: //www.eia.gov/electricity/data/browser/

[4] California Public Utility Commission, "Inputs \& Assumptions: 2019-2020 Integrated Resource Planning," Feb. 2020. Accessed: Jun. 01, 2020. [Online]. Available: https://bit.1y/2Xujudi

[5] Energy + Environmental Economics (E3), RESOLVE model 2020. Accessed: Jun 01, 2020. [Online]. Available: https://bit.ly/36ZNLLV.

[6] California Public Utility Commission, "2019-2020 Electric Resource Portfolios to inform Integrated Resource Plans and Transmission Planning,” 331772681, Apr. 2020. Accessed: Jun. 01, 2020. [Online]. Available: https://bit.ly/3eGqGRg.

[7] R. Bailey, P. A. Sánchez-Pérez, Z. Mahmud and S. Kurtz, "Method for Quantifying Value of Storage Toward reaching 100\% Renewables Electricity," in Proc. IEEE 47th PVSC, 2020. 